\title{
The $m$-Derivations of Distribution LieAlgebras
}

\section{Princy Randriambololondrantomalala*}

Département de Mathématiques et Informatique, Faculté des Sciences, Universitéd'Antananarivo, Antananarivo 101, Madagascar

\begin{abstract}
Let $M$ be a $N$-dimensional smooth differentiable manifold. Here, we are going to analyze $(m>1)$-derivations of Lie algebras relative to an involutive distribution on subrings of real smooth functions on M. First, we prove that any $(m>1)$-derivations of a distribution $\Omega$ on the ring of real functions on $M$ as well as those of the normalizer of $\Omega$ are Lie derivatives with respect to one and only one element of this normalizer, if $\Omega$ doesn't vanish everywhere. Next, suppose that $N=\mathrm{n}+\mathrm{q}$ such that $\mathrm{n}>0$, and let $S$ be a system of $q$ mutually commuting vector fields. The Lie algebra of vector fields $\mathfrak{A}_{S}$ on $\mathrm{M}$ which commutes with $S$, is a distribution over the ring $F_{0}(\mathrm{M})$ of constant real functions on the leaves generated by $S$. We find that $m$-derivations of $\mathfrak{A}_{S}$ is local if and only if its derivative ideal coincides with $\mathfrak{A}_{S}$ itself. Then, we characterize all non local m-derivation of $\mathfrak{A}_{S}$. We prove that all $m$-derivations of $\mathfrak{A}_{s}$ and the normalizer of $\mathfrak{A}_{s}$ are derivations. We will make these derivations and those of the centralizer of $\mathfrak{A}_{S}$ more explicit.
\end{abstract}

AMS Subject Classification: Primary 17B66, 17B40, Secondary 53C12, 53B15, 47B47, 53B40.

Keywords: $m$-derivations; Vector fields lie algebras; Distributions; Commuting vector fields; Generalized foliations; Compactly supported vector fields; $\mu$-projected vector fields; Nullity space of curvature

\section{Introduction and Preliminary}

Let $m$ be a natural integer greater than or equal to 2 . We recall that a $m$-derivation $D$ of a Lie $\mathbb{R}$-algebra $\mathfrak{A}$ is an endomorphism of $\mathfrak{A}$, such that for all $X_{1}, X_{2}, \ldots, X_{m} \in \mathfrak{A}$

$$
\begin{aligned}
& D\left[X_{1},\left[X_{2}, \ldots,\left[X_{m-1}, X_{m}\right] \ldots\right]\right]=\left[D\left(X_{1}\right),\left[X_{2}, \ldots\left[X_{m-1}, X_{m}\right] \ldots\right]\right]+ \\
& +\left[X_{1},\left[D\left(X_{2}\right), \ldots,\left[X_{m-1}, X_{m}\right] \ldots\right]\right]+\ldots+ \\
& +\left[X_{1},\left[X_{2}, \ldots,\left[D\left(X_{m-1}\right), X_{m}\right] \ldots\right]\right]+ \\
& +\left[X_{1},\left[X_{2}, \ldots,\left[X_{m-1}, D\left(X_{m}\right)\right] \ldots\right]\right] .
\end{aligned}
$$

This map is inner with respect to Lie algebra $\mathfrak{B}$ if $D$ equals to a Lie derivative with respect to $X \in \mathfrak{B}$; if $X \in \mathfrak{A}$, it is an inner $m$-derivation. A standard $m$-derivation $D$ is a sum of derivations of $\mathfrak{A}$ and $\mathbb{R}$-linear maps of $\mathfrak{A}$ into the center of $\mathfrak{A}$ such that $D[\mathfrak{A},[\mathfrak{A},[\ldots,[\mathfrak{A}, \mathfrak{A}] \ldots]]=\{0\}$.

Is it sufficient to study derivation of Lie algebras? What is the reason for studying the more general notion:" $(m>2)$-derivation"? In other words, can we find $(m>2)$-derivations of a vector fields Lie algebra which are not derivations? In [1], we found $m$-derivations all polynomial vector fields Lie algebras $P$ on $\mathbb{R}^{n}$, where $P$ contains Euler vector fields $E$ and all constant vector fields. We remark that all these $m$-derivations are derivations when $\mathrm{m}$ is even. If $m$ is an odd number, $m$-derivations are generally sum of derivations and $m$-derivations with homogeneous degree -2 . Over $\mathbb{R}^{3}$, we can take a simple example where the Lie $\mathbb{R}$-algebra is spanned by $\frac{\partial}{\partial x}, \frac{\partial}{\partial y}, \frac{\partial}{\partial z}, E, z \frac{\partial}{\partial x},(z)^{2} \frac{\partial}{\partial x}$ and the $\mathbb{R}$ linear map $D$ is defined by $D\left((z)^{2} \frac{\partial}{\partial x}\right)=\frac{\partial}{\partial x}$ and vanishing otherwise. It is a 3-derivation, but not a derivation. In [2], some graded Lie algebra $m$-derivations are discussed. Here, we are interested in $m$-derivations of distribution Lie algebra on a $\mathrm{N}$-smooth manifold $\mathrm{M}$ over an $\mathrm{M}$-real functions ring. We know that all smooth vector fields can be locally approximated to polynomial vector fields, so we think that all results in [1] are naturally true in the case of distributions. But, the results which follow are different. The differential operator theory see [3] is the main tool throughout our proofs.

We denote by $F(\mathrm{M})$ the ring of all real functions on $\mathrm{M}, \chi(\mathrm{M})$ (resp. $\chi(\mathrm{TM}))$ the vector fields Lie algebras over $\mathrm{M}$ (resp. over the tangent bundle TM).

At first, we consider an involutive distribution $\Omega$ over $F(\mathrm{M})$. That is to say, $\Omega$ is a $F(\mathrm{M})$-sub-module of the module of all vector fields on $\mathrm{M}$. Assuming that the open set $O_{\Omega}=\{x \in \mathrm{M} / \Omega(x) \neq\{0\}\}$ equals $\mathrm{M}$, we are looking for characteristics of $m$-derivations of Lie algebras relative to $\Omega$ and applications of the obtained results on some remarkable distributions. We propose to prove that each $m$-derivation of $\Omega$ (resp. of the normalizer in $\chi(\mathrm{M})$ of $\Omega$ ) is simply a Lie derivative with respect to one and only one normalizer's vector fields (resp. is inner). These theorems can be extended where $O_{\Omega}$ is dense over $\mathrm{M}$.

Secondly, let be $N=n+q$ with $n \geq 1$ and $q>0, S$ a system of $q$ nonvanishing vector fields which commute mutually. We know by results in [4] that $S$ yields a generalized foliation on $\mathrm{M}$. We assume that all leaves are regular and we notice that $F_{0}(\mathrm{M})$, the ring of real smooth functions which are constant on the leaves over M. Let $U$ be a $p$-dimensional adapted chart domain relative to the foliation and $\left(x^{a}, y^{i}\right)$ (resp. $\left(U, x^{a}\right)$ ), where $1 \leq a \leq n+q-p, 1 \leq i \leq p$ if $p \geq 1$ (resp. where $1 \leq a \leq n+q$ if $\mathrm{p}=0$ ). Then, there are two modules over $F_{0}(U), A_{S}^{1}(U)$ spanned by $\left(\frac{\partial}{\partial x^{a}}\right)_{1 \leq a \leq n+q-p}$ and $\mathfrak{A}_{S}^{2}(U)$ generated by $\left(\frac{\partial}{\partial y^{i}}\right)_{1 \leq i \leq p}$. These previous modules are Lie algebras such

*Corresponding author: Princy Randriambololondrantomalala, Département de Mathématiques et Informatique, Faculté des Sciences, Universitéd'Antananarivo, Antananarivo 101, BP 906, Madagascar, Tel: +261 2022326 39; E-mail: Princypcpc@yahoo.fr

Received November 14, 2014; Accepted March 07, 2015; Published March 16 2015

Citation: Randriambololondrantomalala $\mathrm{P}$ (2015) The $m$-Derivations of Distribution LieAlgebras. J Generalized Lie Theory Appl 9: 217. doi: 10.4172/17364337.1000217

Copyright: (c) 2015 Randriambololondrantomalala P. This is an open-access article distributed under the terms of the Creative Commons Attribution License, which permits unrestricted use, distribution, and reproduction in any medium, provided the original author and source are credited. 
that $\mathfrak{A}_{S}(U)$ is equal to the semi-direct product of these two algebras: $\mathfrak{A}_{S}(U)=\mathfrak{A}_{S}^{1}(U) \oplus \mathfrak{A}_{S}^{2}(U)$ for all distinguished $U$. We can say that $\mathfrak{A}_{S}$ is a smooth distribution of $\mathrm{M}$ over $F_{0}(\mathrm{M})$. Throughout this paper, we assume that this chart is $(p>0)$-dimensional in the sense of the foliation, unless expressly stated. Our aims are to characterize all m-derivations of $\mathfrak{A}_{S}$, of the normalizer of $\mathfrak{A}_{S}$ and of the centralizer of $\mathfrak{A}_{S}$ in $\chi(\mathrm{M})$. The corresponding work where $S=\{0\}$ has been done in the previous section. Because of $\mathfrak{A}_{S}$ 's lower central series constancy, which coincides with module direct sum of $\mathfrak{A}_{S}^{1} \oplus\left[\mathfrak{A}_{s}^{1}, \mathfrak{A}_{S}^{2}\right]$, our work on these $m$-derivations is non-trivial. The main results of this section are: all $m$-derivation is local iff the derivative ideal of $\mathfrak{A}_{S}$ is $\mathfrak{A}_{S}$ itself, which is equivalent to the fact that $\mathfrak{A}_{S}^{1}\left(F_{0}(\mathrm{M})\right)$ has non-vanishing elements over the whole $M$. Moreover, all $m$-derivations of $\mathfrak{A}_{s}$ or of the normalizer $\mathfrak{N}$ of $\mathfrak{A}_{S}$ are sums of a Lie derivative with respect to one $\mathfrak{N}$ 's element, of one local $m$-derivation which takes its value in $\mathfrak{A}_{S}^{2}$ depending on two non-vanishing 1-differential forms over $\mathfrak{A}_{S}$, and of a non-local m-derivation of $\mathfrak{A}_{S}$. We give some recommendations for constructing all these non local $m$-derivations. In addition, all $\mathbb{R}$ -linear maps of $\langle S\rangle$ the centralizer of $\mathfrak{A}_{S}$ into itself are $m$-derivations. We characterize all local $\mathbb{R}$-endomorphisms of $\langle S\rangle$ in the case where all elements of $S$ are densely supported or $\langle S\rangle$ is spanned by singleton, and those which are non local. It is well known that the open set of all foliation regular points is dense in $\mathrm{M}$, then one can extend these results where the foliation is singular and if the above 1-forms prolongs smoothly on $\mathrm{M}$.

Several applications of our results about Lie algebras relative to: all vector fields, all compactly supported vector fields, generalized foliations, $\mu$-projected vector fields cf. [5], $k$-nullity space of connection curvature, and vector fields Lie algebras on TM commuting with Liouville vector fields cf. [6]; are given at the end of this paper.

Throughout this article, the Lie derivative with respect to $X \in \chi(\mathrm{M})$ is denoted $\mathrm{L}_{x}$. We adopt the Einstein index summation and suppose that all considered objects are smooth.

\section{The $\boldsymbol{m}$-derivations of Lie algebras attached to $\Omega$}

According the hypothesis about $\Omega$, we can affirm that $\Omega$ is a Lie sub-algebra of $\chi(M)$. A generalization of $[7,8]$ 's theorems in the sense of derivation or triple derivation can be stated as follows:

Theorem 2.1. All m-derivations of $\Omega$ (resp. of the normalizer of in $\chi(\mathrm{M})$ ) are Lie derivative with respect to one and only one vector field of the normalizer of $\Omega$ (resp. is inner).

Proof. Assume that $x \in \mathrm{M}, \exists X \in \Omega$ such that $X(x) \neq 0$. By Frobenius theorem, we find one chart $\left(U_{x}, \varphi_{x}\right.$, which contains $x$ and local coordinate system $\left(x^{1}, \ldots, x^{n-1}, y\right)$ where $X_{U_{x}} \frac{\partial}{\partial y}$. Letting $D$ be an $m$-derivation of $\Omega$, we know that the Lie algebra spanned by brackets of all elements in $\Omega$ is the derivative ideal of $\Omega$ denoted by [ $\Omega$; $\Omega$ ]. Local behavior of $D$ can be proved by adapting one of Proposition 2.4 in [7] and using that the derivative ideal of $\Omega$ is $\Omega$ itself. Therefore $D_{U_{r}}$ is an $m$-derivation of $\Omega_{U_{x}}$. Let's give $f \in F\left(U_{x}\right)$, as we know, $\frac{\partial}{\partial y} \in \Omega_{U_{x}}$ then $D_{U_{x}}\left(f \frac{\partial}{\partial y}\right)=D^{0}(f) \frac{\partial}{\partial y}+D_{0<a<n}^{a}(f) \frac{\partial}{\partial x^{a}}$ is uniquely determined, where each $D^{i}$ is differential operator over the trivial bundle $U_{x} \times \mathbb{R}$ cf. [3]. Thus, if necessary we can write $D^{0}=\sum_{|A| 0,0,|B| 1, q \geq 1, r \geq 1} \chi^{A, 0} \frac{\partial^{|A|}}{\partial x^{A}}+\chi^{B, q} \frac{\partial^{|B|+q}}{\partial x^{B} \partial y^{q}}+\chi^{0, r} \frac{\partial^{r}}{\partial y^{r}}$, where $A, B$ are multi-indices corresponding to coordinates.

Let's apply $D_{U_{x}}$ to $\left[x^{j} \frac{\partial}{\partial y},\left[y \frac{\partial}{\partial y}, \ldots,\left[y \frac{\partial}{\partial y}, f \frac{\partial}{\partial y}\right] \ldots\right]\right]$, where $x^{n}=y$. By definition of $m$-derivations and when $f$ is replaced by monomials, we have:

$$
\begin{aligned}
& \text { If } \operatorname{deg}(f) \geq 2, D^{0}(f)(0, \ldots, 0)=0 \text { except for } f \equiv y^{2} . \\
& \text { If } \operatorname{deg}(f) \geq 1, D^{a}(f)(0, \ldots, 0)=0 \text { except for } f \equiv y P\left(x^{i}, i \neq n\right)
\end{aligned}
$$

where $P\left(x^{i}\right)$ are free $x^{n}$ monomials.

By reasoning as in the previous, we compute $D_{U_{x}}\left[\frac{\partial}{\partial y},\left[\frac{\partial}{\partial y}, \ldots,\left[\frac{\partial}{\partial y}, y^{m} P\left(x^{i}, i \neq n\right) \frac{\partial}{\partial y}\right] \ldots\right]\right]$. It is easy, using both the previous relation and the previous proof, to obtain the nullity of $D^{a}\left(y P\left(x^{i}\right)\right)(0, \ldots, 0)$. By coordinates translations, we can affirm that each $D^{j}, j \neq 0$ is a differential operator of order 0 and $\mathrm{D}^{0}$ is a sum of one of order 1 with one other $\chi^{0,2} \frac{\partial^{2}}{(\partial y)^{2}}$ of order 2 .

Computing in the same way as the previous calculus, $D_{U_{x}}\left[f \frac{\partial}{\partial y},\left[y \frac{\partial}{\partial y}, \ldots,\left[y \frac{\partial}{\partial y}, \frac{\partial}{\partial y}\right] \ldots\right]\right]$ gives:

- for $f \equiv y, D^{0}(1)=-\frac{\partial \chi^{0,1}}{\partial y}$.

- for $f \equiv x^{j}$ except $j \neq n, D^{j}(1)=-\frac{\partial \chi^{\left(0, \ldots, 0,1_{j}, 0, \ldots, 0\right)}}{\partial y}\left(1_{j}\right.$ means 1 is in $j$-th rank).

By these results, $D_{U_{x}}\left(f \frac{\partial}{\partial y}\right)=\mathrm{L}_{\chi^{0,1} \frac{\partial}{\partial y}+\chi^{\left(0, \ldots, 0, l_{a}, 0, \ldots, 0\right)}}\left(f \frac{\partial}{\partial x^{a}}(f y)+\chi^{0,2} \frac{\partial^{2} f}{(\partial y)^{2}} \frac{\partial}{\partial y}\right.$. Consequently,

$D_{0}=\chi^{0,2} \frac{\partial^{2}}{(\partial y)^{2}} \otimes \frac{\partial}{\partial y}$ is a derivation of the $F\left(U_{x}\right)$-sub-module spanned by $\frac{\partial}{\partial y}$. Applying $D_{0}$ to $\left[y^{2} \frac{\partial}{\partial y},\left[\frac{\partial}{\partial y}, \ldots,\left[\frac{\partial}{\partial y}, y^{m-1} \frac{\partial}{\partial y}\right] \ldots\right]\right]$, we have $\chi^{0,2}(y=0)=0$. By coordinate's translations, we can write that $\chi^{0,2}=0$.

We take Proposition 2.6 of [7] and we have $D_{U_{x}}=\mathrm{L}_{\chi^{0,1} \frac{\partial}{\partial y}+\chi^{\left(0, \ldots, \alpha_{a}, 0, \ldots, 0\right)} \frac{\partial}{\partial x^{a}}}$. Follwing the arguments of the proof of Theorem 2.7 in [7], we end the demonstration of the first assertion of our theorem. Taking that the derivative ideal of $\Omega$ is $\Omega$ itself into account, we can adapt the proof of Theorem 2.12 in [7] to state the second assertion.

Remark 2.2. These theorems are correct if we consider $O_{\Omega}$ to be dense over $\mathrm{M}$ and if the corresponding vector of the Lie derivative relative to the $m$-derivation cited by Theorem 2.1 can be smoothly extended towards $\mathrm{M}$.

\section{The $\boldsymbol{m}$-derivations of Lie algebras defined by $\mathfrak{A}_{s}$}

We know that nil potency of order $m-1$ of $\mathfrak{A}_{S}$ forces any endomorphism of $\mathfrak{A}_{S}$ to be an $m$-derivation. To avoid this triviality, we prove that:

Proposition 3.1. The lower central series of $\mathfrak{A}_{S}$ are constant and equal to the module $\mathfrak{A}_{S}^{1} \oplus\left[\mathfrak{A}_{s}^{1}, \mathfrak{A}_{S}^{2}\right]$.

Proof. The lower central series of $\mathfrak{A}_{S}$ is determined by $\mathfrak{C}\left(\mathfrak{A}_{S}\right) \quad \mathfrak{A}_{S}$ and for all $p>0$,

$\mathfrak{C}^{p}\left(\mathfrak{A}_{S}\right)=\left[\mathfrak{A}_{S}, \mathfrak{C}^{p-1}\left(\mathfrak{A}_{S}\right)\right]$ cf.[1]. By Proposition 3.7 of [4], the derivative ideal of $\mathfrak{A}_{S}$ is $\mathfrak{A}_{S}^{1} \oplus\left[\mathfrak{A}_{S}^{1}, \mathfrak{A}_{S}^{2}\right]$. From the linearity of brackets, the Jacobi identity and the fact that $\mathfrak{A}_{S}^{2}$ is an ideal of $\mathfrak{A}_{S}$, we deduce $\left[\mathfrak{A}_{S},\left[\mathfrak{A}_{S}^{1} \oplus\left[\mathfrak{A}_{S}^{1}, \mathfrak{A}_{S}^{2}\right]\right]\right]=\mathfrak{A}_{S}^{1} \oplus\left[\mathfrak{A}_{S}^{1}, \mathfrak{A}_{S}^{2}\right]$. Then, we deduce the result.

We assume the following conventions about the index, 
$i, j, k \in\{1, \ldots, p\}, a, b, c \in\{1, \ldots, n+q-p\}$, and each index indexed by 0 is fixed.

Proposition 3.2. Let $D$ be a m-derivation of $\mathfrak{A}_{S}$ and $U$ a domain of distinguished chart such that if $X \in \mathfrak{A}_{S}$ over $U$ vanishes, then $D(X)$ over $U$ on $\mathfrak{A}_{S}^{1}$ is zero.

Proof. Let $D$ be a such $m$-derivation and $X$ an element of $\mathfrak{A}_{S}$ satisfying the above hypothesis. We assume that $D(X)_{\mid A_{S}^{1}} \neq 0$, then it exists an open set $V_{z}$ containing $z$, such that the $a_{0}$-th component of $D(X)_{\mid A_{s}^{\prime}}$ on $V$ is everywhere non zero. Let's consider $f \in F_{0}(\mathrm{M})$ such that $f_{V_{z}}=\left(x^{a_{0}}\right)^{2}$ where $\operatorname{Supp}(f) \subset U$, and $Y, X_{3}, \ldots, X_{m}$ are elements of $\mathfrak{A}_{S}$ with $Y_{\mid V_{z}}=\frac{\partial}{\partial y^{i_{0}}}, X_{3 \mid V_{z}}=\ldots=X_{m-1 \mid V_{z}}=x^{a_{0}} \frac{\partial}{\partial x^{a_{0}}}, X_{m \mid V_{z}}=\frac{\partial}{\partial x^{a_{0}}}$. By definition, we obtain

$$
D\left[X,\left[f Y,\left[X_{3}, \ldots\left[X_{m-1}, X_{m}\right] \ldots\right]\right]\right](z)=\left[D(X),\left[f Y,\left[X_{3}, \ldots\left[X_{m-1}, X_{m}\right] \ldots\right]\right]\right](z)+[X, Z](z)
$$

With $Z \in \mathfrak{A}_{S}$, a contradiction.

Proposition 3.3. The centralizer $\mathcal{C}$ of $\mathfrak{A}_{S}^{1} \oplus\left[\mathfrak{A}_{S}^{1}, \mathfrak{A}_{S}^{2}\right]$ coincides with the vector $\mathbb{R}$-space $\langle S\rangle$ spanned by $S$.

$$
\begin{aligned}
& \text { Proof. Recall that } \\
& \mathcal{C}=\left\{X \in \chi(\mathrm{M}) /\left[X, \mathfrak{A}_{S}^{1} \oplus\left[\mathfrak{A}_{S}^{1}, \mathfrak{A}_{S}^{2}\right]\right]=\{0\}\right\} .
\end{aligned}
$$

Choose $X \in \mathcal{C}$ and let be $U$ a distinguished connected chart domain of the foliation. When $p=0$, we have $X=0$. For $p \geq 1$, we put $X_{\mid U}=X^{a} \frac{\partial}{\partial x^{a}}+X^{\prime i} \frac{\partial}{\partial y^{i}} \in \mathfrak{C}_{U}$. By the fact $\left[X, \mathfrak{A}_{S}^{1}\right]=\{0\}, X^{a}=0$ for all $a$ and each $X^{\prime i} \in\left(F(U)-F_{0}(U)\right) \cup\left(F(U) \cap F_{0}(U)\right)$. Therefore, $X_{|2|_{s}}=0$ and $X=f^{i} X_{i}$ where all $f^{i} \in\left(F(\mathrm{M})-F_{0}(\mathrm{M})\right) \cup\left(F(\mathrm{M}) \cap F_{0}(\mathrm{M})\right)$. Assume $Y^{1}\left(g^{i}\right) X_{i} \in\left[\mathfrak{A}_{S}^{1}, \mathfrak{A}_{S}^{2}\right]$ with $g^{j} X_{j} \in \mathfrak{A}_{S}^{2}, Y^{1} \in \mathfrak{A}_{S}^{1}$. It's known that $\left[X, \mathfrak{A}_{S}^{1} \oplus\left[\mathfrak{A}_{S}^{1}, \mathfrak{A}_{S}^{2}\right]\right]=\{0\}$ so $Y^{1}\left(g^{i}\right) X_{i}\left(f^{j}\right) X_{j}=0$ for all $g^{i}$ and $Y^{1}$. Then all $f^{j}$ are in $F_{0}(\mathrm{M})$ and consequently they are constant, and $\mathcal{C}$ is a subset of the $\mathbb{R}$-vector space spanned by $S$. The converse inclusion obvious.

Proposition 3.4. All non-local m-derivations of $\mathfrak{A}_{S}$ vanish on $\mathfrak{A}_{S}^{1} \oplus\left[\mathfrak{A}_{S}^{1}, \mathfrak{A}_{S}^{2}\right]$ and take their values in $\langle S\rangle$. Conversely, all $\mathbb{R}$ -endomorphisms D of $\mathfrak{A}_{S}$ which have these properties, is a m-derivation of $\mathfrak{A}_{S}$. All theses maps are standard m-derivations.

Proof. To simplify, we pose a such $m$-derivation $D$. Then there is $X \in \mathfrak{A}_{S}$ and a distinguished chart domain $U$ so that $X_{\mid U} \equiv 0$ with $D(X)(z \in U) \neq 0$. Thus, we have an open set $V_{z}$ in $U$ containing $z$, with $D(X)$ everywhere non-vanishing. Recall that the center of $\mathfrak{A}_{S}$ is the intersection of its centralizer with itself. We reason by contradiction, we suppose that $D(X)$ doesn't belong to the center of $\mathfrak{A}_{S}$. By Proposition 3.2, we claim that on $V$, the $i_{0}$-th component of $D(X)$ is everywhere non vanishing. So, this component is not a constant function. Consequently, we can assume that its partial derivative with respect to a $x^{a_{0}}$ is non-zero at $z$. Then, we consider $X_{2}, \ldots, X_{m}$ to be elements of $\mathfrak{A}_{S}$ such that $\operatorname{Supp}\left(X_{2}\right) \subset U$ and $X_{2 \mid V_{z}}=\ldots=X_{m-1 \mid V_{z}}=x^{a_{0}} \frac{\partial}{\partial x^{a_{0}}}, X_{m \mid V_{z}}=\frac{\partial}{\partial x^{a_{0}}}$. By the $m$-derivation definition,

$$
D\left[X,\left[X_{2},\left[X_{3}, \ldots\left[X_{m-1}, X_{m}\right] \ldots\right]\right]\right](z)=\left[D(X),\left[X_{2},\left[X_{3}, \ldots\left[X_{m-1}, X_{m}\right] \ldots\right]\right]\right](z)+[X, Z](z)
$$

Where $Z \in \mathfrak{A}_{S}$, we have a contradiction. Moreover, Proposition
3.1 and the previous result lead to nullity of $D$ over $\mathfrak{A}_{S}^{1} \oplus\left[A_{S}^{1}, \mathfrak{A}_{S}^{2}\right]$ It is easy to prove the last assertions of our proposition.

We can note immediately that,

Lemma 3. For all $k \geq 2$, if $D$ is a $k$-derivation of Lie algebra $\mathfrak{A}$ then the center $\mathrm{C}$ of $\mathfrak{A}$ satisfies the following equation $\left[D(\mathrm{C}), \mathfrak{C}^{k-2}(\mathfrak{A})\right]=\{0\}$.

Proposition 3.6. Local m-derivations of $\mathfrak{A}_{S}$ stabilize $\mathfrak{A}_{S}^{2}$.

Proof. We set a local $m$-derivation $D, D_{\mid U}$ is still an $m$-derivation. Without trivial case $p=0$, let $a, b, i$ be some fixed indices, we write

$D_{U}\left(x^{b} \frac{\partial}{\partial y^{i}}\right)=D_{i, b}^{c} \frac{\partial}{\partial x^{c}}+D_{i, b}^{j+m+q-p} \frac{\partial}{\partial y^{j}}$

And

$(-1)^{m-1} \delta_{a}^{b} D_{U}\left(\frac{\partial}{\partial y^{i}}\right)=D_{U}\left(\left[x^{b} \frac{\partial}{\partial y^{i}},\left[x^{a} \frac{\partial}{\partial x^{a}},\left[\ldots,\left[x^{a} \frac{\partial}{\partial x^{a}}, \frac{\partial}{\partial x^{a}}\right] \ldots\right]\right]\right]\right), \forall a$.

By using the (3.2), Lemma 3.5 and Proposition 3.3, we deduce that each $D_{i, b}^{c}$ is constant.

Let $f$ be an element of $F_{0}(U)$,We remark that

$$
\left[f \frac{\partial}{\partial x^{b}},\left[x^{c} \frac{\partial}{\partial x^{c}},\left[\ldots,\left[x^{c} \frac{\partial}{\partial x^{c}}, x^{b} \frac{\partial}{\partial y^{i}}\right] \ldots\right]\right]\right]=f \frac{\partial}{\partial y^{i}} .
$$

By mapping $D_{U}$ to (3.3) in the case where $f$ is a polynomial of degree greater or equal than two, the previous result and the fact that $D_{i, b}^{c}$ is a differential operator over $U \times \mathbb{R}$, proves that $D_{U}\left(f \frac{\partial}{\partial y^{i}}\right) \in \mathfrak{A}_{S}^{2}(U)$ for all $i$. Furthermore, combining the previous results and the obtained relation by

$$
D_{U}\left[\left(x^{b}\right)^{2} \frac{\partial}{\partial x^{b}},\left[x^{c} \frac{\partial}{\partial x^{c}},\left[\ldots,\left[x^{c} \frac{\partial}{\partial x^{c}}, \frac{\partial}{\partial x^{b}}\right] \ldots\right]\right]=-2 D_{U}\left(x^{b} \frac{\partial}{\partial y^{i}}\right)\right.
$$

We see that $D_{i, b}^{c}=0$. Then, $D_{U}\left(x^{b} \frac{\partial}{\partial y^{i}}\right) \in \mathfrak{A}_{S}^{2}(U)$

In addition,

$$
D_{U}\left(f \frac{\partial}{\partial y^{i}}\right)=D_{U}\left[f \frac{\partial}{\partial x^{b}},\left[x^{c} \frac{\partial}{\partial x^{c}},\left[\ldots,\left[x^{c} \frac{\partial}{\partial x^{c}}, x^{b} \frac{\partial}{\partial y^{i}}\right] \ldots\right]\right]\right]
$$

and the previous statement leads to $D_{U}\left(f \frac{\partial}{\partial y^{i}}\right) \in \mathfrak{A}_{S}^{2}(U)$ for all $U$.

Proposition 3.7. The Lie algebra $\mathfrak{A}_{S}^{2}$ is stabilized by m-derivations of $\mathfrak{A}_{S}$.

Proof. We deduce the result from Propositions 3.4, 3.6.

Theorem 3.8. We have equivalences between:

1. All m-derivation of $\mathfrak{A}_{S}$ is local.

2. There is an $X \in A_{S}^{1}$ and $h \in F_{0}(\mathrm{M})$ such that $X(h)(x) \neq 0 \forall x \in \mathrm{M}$.

3. The derivative ideal of $\mathfrak{A}_{S},\left[\mathfrak{A}_{S}, \mathfrak{A}_{S}\right]$ coincides with $\mathfrak{A}_{S}$ itself.

Proof. In $2 . \Rightarrow 1$., we use the same reasoning as the one of the proof of Theorem 3.11 in [4]. As for $1 . \Rightarrow 2$. we suppose that there is an $f \notin F_{0}(\mathrm{M}) \mathfrak{A}_{s}^{1}\left(F_{0}(\mathrm{M})\right)$. Since $S \neq 0$, then it exists $k$ such that $X_{k}$ is nonzero on the open set $U_{k}$, and $g \in F_{0}(\mathrm{M})$ vanishing on $U_{k}$ with $(f g)_{\mid U_{k}} \equiv 0$. So, it is immediate that the $\mathbb{R}$-linear map defined by 


$$
D(X)= \begin{cases}0 & \text { ifX } \in \mathfrak{A}_{\mathrm{s}}-\left\{\mathbb{R f g X}_{\mathrm{k}}\right\}, \\ D^{j} X_{j} & \text { ifX }=\mathrm{fgX}_{\mathrm{k}} \text { whereD }^{\mathrm{j}} \in \mathbb{R} \text { forallj }=1, \ldots, \mathrm{q} .\end{cases}
$$

Is a non-local $m$-derivation when $D^{k} \neq 0$. Thus 1 . $\Leftrightarrow 2$. .

We reason in the same way as in [4] for 1 . $\Leftrightarrow 3$..

Remark 3.9. We assert that if the derivative ideal of $\mathfrak{A}_{S}$ doesn't coincide with $\mathfrak{A}_{S}$, then it exists $f \notin F_{0}(\mathrm{M}) \mathfrak{A}_{S}^{1}\left(F_{0}(\mathrm{M})\right)$, zero on the open set where one $X_{k}$ is non-vanishing. To realize a non local $m$-derivation $D$, we exploit the non-vanishing on $\mathfrak{A}_{S}-\left(\mathfrak{A}_{s}^{1} \oplus\left[\mathfrak{A}_{S}^{1}, \mathfrak{A}_{S}^{2}\right]\right)$ of the following $\mathbb{R}$-linear map:

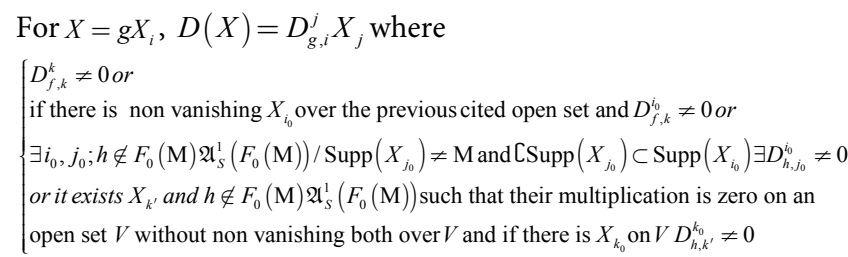

These results are immediate by using Theorem 3.8, Proposition 3.4 and the definition of non local $\mathfrak{A}_{S}$ m-derivation.

Proposition 3.10. The normalizer $\mathfrak{N}$ of $\mathfrak{A}_{S}$ in $\chi(\mathrm{M})$ is locally isomorphic to $\mathfrak{A}_{s} \oplus g l(p, \mathbb{R})$ as a vector space, where $p$ is the corresponding leaf local dimension. So $\left[\mathfrak{N}, \mathfrak{A}_{S}\right]=\mathfrak{A}_{S}$ locally $\left[\mathfrak{N}, \mathfrak{A}_{s}^{1} \oplus\left[\mathfrak{A}_{s}^{1}, \mathfrak{A}_{s}^{2}\right]\right]=\mathfrak{A}_{s}$. Moreover, all local m-derivations of $\mathfrak{N}$ stabilize $\mathfrak{A}_{S}$

Proof. We define $\mathfrak{N}$ by the set of all vector fields $X$ such that $\left[X, \mathfrak{A}_{S}\right] \subset \mathfrak{A}_{S}$. So, we are in a distinguished chart $U$, all $\mathfrak{N}_{U}$ ' $s$ elements are obtained with direct use of the definition of the normalizer of $\mathfrak{A}_{S}(U)$.Indeed, $\mathfrak{N}_{\mid U}$ is the sum of $\mathfrak{A}_{S}(U)$ and the vector $\mathbb{R}$-space spanned by $y^{i} \frac{\partial}{\partial y^{j}}$. It's clear that, this last space is isomorphic to $g l(p, \mathbb{R})$. The two results which follow are easily proved by the same argument as the previous. As for the last assertion, let's take $X_{1} \in \mathfrak{N}$, $X_{2}, \ldots, X_{m} \in \mathfrak{A}_{S}$ and $D$ a local $m$-derivation of $\mathfrak{N}$. In accordance with the $m$-derivation definition, we have $D\left[X_{1},\left[X_{2}, \ldots,\left[X_{m-1}, X_{m}\right] \ldots\right]\right] \in \mathfrak{A}_{s}$. By local equation $\left[\mathfrak{N}, \mathfrak{A}_{S}^{1} \oplus\left[\mathfrak{A}_{S}^{1}, \mathfrak{A}_{S}^{2}\right]\right]=\mathfrak{A}_{S}$, Proposition 3.1 and the previous result where each $X_{i}$ runs through over the respective sets, we affirm that $D_{\mid \mathfrak{A l}_{S}}\left(\mathfrak{A}_{S}\right)$ is subset of $\mathfrak{A}_{S}$.

Theorem 3.11. Given that we have a local m-derivation $D$ of $\mathfrak{A}_{S}$ towards $\mathfrak{A}_{S}^{2}$. We find 1 differential closed forms $\alpha^{i}$ and $\omega^{i}$ over $U$, where $i=1, \ldots, p$ with $D_{U}=\left(\alpha^{j}+\omega^{j}\right) \otimes \frac{\partial}{\partial y^{j}}$ denoted $D_{U}^{(\alpha, \omega)}$ such that $\operatorname{ker}\left(\alpha^{j}\right) \supset \mathfrak{A}_{S}^{2}(U)$ and $\operatorname{ker}\left(\omega^{j}\right) \supset \mathfrak{A}_{S}^{1}(U)$. Besides,

$$
\alpha^{i}\left[X_{1}, X_{2}\right]=X_{1} \cdot \alpha^{i}\left(X_{2}\right)-X_{2} \cdot \alpha^{i}\left(X_{1}\right)
$$

$\forall X_{1}, X_{2} \in \mathfrak{A}_{S}(U)$. The converse of this result is also true. Furthermore, the condition that the maps $D_{U}^{(\alpha, \omega)}$, with $\alpha=\left(\alpha^{1}, \ldots, \alpha^{p}\right)$ and $\omega=\left(\omega^{1}, \ldots, \omega^{p}\right)$, are inner is equivalent to, for all $i, \omega^{i} \equiv 0$ and $\alpha^{i}$ are exact. Then we get $D_{U}^{(\alpha, \omega)}=-\mathrm{L}_{f^{i}} \frac{\partial}{\partial i^{i}}$ Where, $\alpha^{i}=d f^{i}$ with $f^{i} \in F_{0}(U)$. Generally if $\alpha=0$, then $D_{U}^{(\alpha, \omega)}=\mathrm{L}_{y^{\prime} C_{i}^{j}} \frac{\partial}{\partial y^{j}}$ where $C_{i}^{j} \in \mathbb{R}$

Proof. Agreeing with the above hypothesis, we pose $D_{U}=\gamma^{i} \otimes \frac{\partial}{\partial y^{i}}$, where the $\gamma^{i}=\gamma_{a}^{i} d x^{a}+\gamma_{j}^{\prime i} d y^{j}$ with $\gamma_{a}^{i}, \gamma_{j}^{\prime i}$ belong to $F_{0}(\mathrm{U})$. By the relations of $m$-derivations which come from

$$
D_{U}\left[\frac{\partial}{\partial y^{j_{0}}},\left[x^{a} \frac{\partial}{\partial x^{a}}, \ldots,\left[x^{a} \frac{\partial}{\partial x^{a}}, \frac{\partial}{\partial x^{a_{0}}}\right] \ldots\right],\right.
$$

we state that $\frac{\partial \gamma_{j_{0}}^{\prime i}}{\partial x_{0}}=0$ for all $j_{0}$ and $a_{0}$. We write the subsequent equality

$$
D_{U}\left[\frac{\partial}{\partial x^{a_{0}}},\left[x^{a} \frac{\partial}{\partial x^{a}}, \ldots,\left[x^{a} \frac{\partial}{\partial x^{a}}, \frac{\partial}{\partial x^{b_{0}}}\right] \ldots\right] \text { at }(0, \ldots, 0) .\right.
$$

Then, we can have $\left(\frac{\partial \gamma_{a_{0}}^{i}}{\partial x^{b_{0}}}-\frac{\partial \gamma_{b_{0}}^{i}}{\partial x^{a_{0}}}\right)(0, \ldots, 0)=0$, for all $a_{0}, b_{0}$. So, with the help of coordinate's translations, we get the previous equality at other arbitrary points in $U$. Thus, each $\gamma^{i}$ is closed. By exploiting all these assertions, we can adapt the demonstrations of Proposition 3.14, 3.15 et 3.16 of [4] and we achieve our proof.

Let $\Re$ be the set of pair of forms $(\alpha, \omega)$ quoted before. We will denote by $\mathfrak{Z}(U)$, the complement set of those of $(\alpha, \omega) \in \Re$ such that $\alpha$ is exact and $\omega=0$ or $\alpha=0$. We might assume that $\mathfrak{Z}=\mathfrak{Z}(\mathrm{M})$.

Theorem 3.12. The form of m-derivations of $\mathfrak{A}_{S}$ is $\mathrm{L}_{X}+D^{(\alpha, \omega) \in \mathcal{3}}+D_{1}$ where $X \in \mathfrak{N}$, for all distinguished chart $U, D^{(\alpha, \omega)}{ }_{U U}=0$ if the leaf dimension over $U$ is zero; $D^{(\alpha, \omega)}=D_{U}^{(\alpha, \omega) \in 3_{U}}$ otherwise. And $D_{1}$ is a non local $m$-derivation analogous to the one of Remark 3.9. Particularly, these $m$-derivations are derivations.

Proof. Taking $D$ an $m$-derivation of $\mathfrak{A}_{S}$, it is split into a sum of local $m$-derivation $D_{0}$ and of a non-local $m$-derivation $D_{1}$ of $\mathfrak{A}_{S}$. So, $D_{1}$ has the same form as the one of Remark 3.9. We can write $D_{0}$ as $D_{0}^{11}+D_{0}^{12}+D_{0}^{21}+D_{0}^{22}$ with $D_{0}^{l t}$ the $\mathbb{R}$-linear component of $D_{0}$ mapping $\mathfrak{A}_{S}^{l}$ to $\mathfrak{A}_{S}^{t}$, where $l, t=1,2$. By Proposition 3.7, $D_{0}^{21}=0$. In accordance with the same proposition, we can divide $\mathfrak{A}_{S}$ by $\mathfrak{A}_{S}^{2}$ and the quotient $m$ derivation of $D_{0}$ is denoted $\overline{D_{0}}$. The map $\overline{D_{0}}$ becomes an $m$-derivation of $\overline{\mathfrak{A}_{S}^{1}}$ by the splitting of $D_{0}$ we know that $\mathfrak{A}_{S}^{l}$ is locally isomorphic to $\chi\left(\mathbb{R}^{n+q-p}\right)$. Then $\overline{D_{0}}$ coincides with $\mathrm{L}_{X}$ where $X \in \overline{\mathfrak{A}_{S}^{1}}$. Consequently, $D_{0}=\mathrm{L}_{Y}+D^{(\alpha, \omega)}$ with $Y \in \mathfrak{N}$ and $D^{(\alpha, \omega)}$ , $(\alpha, \omega) \in \mathfrak{Z}$ is defined by Theorem 3.11. With the help of Proposition 3.4, respectively Theorem $3.11, D_{1}$ respectively $D_{0}$ is a derivation.

Proposition 3.13. All $m$-derivation of the Lie $\mathbb{R}$-algebra of all linear fields taking value in the constant fields Lie $\mathbb{R}$-algebra of $\mathbb{R}^{t}$ is Lie derivative with respect to one constant field.

Proof. Let $D$ be such $m$-derivation and $\left(z^{l}\right)_{1<l<t}$ one coordinates system of $\mathbb{R}^{t}$. We note that $D\left(z^{u} \frac{\partial}{\partial z^{v}}\right)=D_{v}^{u l} \frac{\partial}{\partial z^{l}}$ with $u, v \in\{1, \ldots, t\}$. It's easy to verify that $D$ vanishes if and only if $D(E)$ is zero too by using the following equation

$$
D\left[E,\left[E, \ldots,\left[E, z^{u} \frac{\partial}{\partial z^{v}}\right] \ldots\right]=\left[E,\left[E, \ldots,\left[E, D\left(z^{u} \frac{\partial}{\partial z^{v}}\right)\right] \ldots\right]\right]\right.
$$

Where $E$ is the Euler vector field. Then, we write $D(E)=C^{l} \frac{\partial}{\partial z^{l}}$. For different and fixed $u, v$, we exploit the obtained relation from

$$
D\left[z^{u} \frac{\partial}{\partial z^{u}},\left[z^{u} \frac{\partial}{\partial z^{u}}, \ldots,\left[z^{u} \frac{\partial}{\partial z^{u}}, z^{u} \frac{\partial}{\partial z^{v}}\right] \ldots\right] .\right.
$$

Therefore, we have $D_{v}^{u l}=0$ where $l \neq u, v ; D_{v}^{u v}=D_{u}^{u u}$. In accordance with (3.4) when $\mathrm{u}=\mathrm{v}$, we state that $C^{u}=D_{u}^{u u}$ and $D_{u}^{u l}=0$ for $l \neq u$. In 
addition, (3.4) gives us $D_{v}^{u u}=0$. Thus, we proved that $D=\mathrm{L}_{C^{\prime}} \frac{\partial}{\partial z^{l}}$.

Proposition 3.14. All m-derivation of $\mathfrak{N}$ taking its value in is a sum of m-derivations of $\mathfrak{A}_{S}$ towards $\langle S\rangle$ and m-derivation of $\left(\mathfrak{N}-\mathfrak{A}_{S}\right) \cup\{0\}$ to $\langle S\rangle$.

Proof. Let $D$ be a $m$-derivation of $\mathfrak{N}$ towards $\langle S\rangle$. It is known that every local $m$-derivation of $\mathfrak{N}$ stabilizes $\mathfrak{A}_{s},\left(\mathfrak{N}-\mathfrak{A}_{s}\right) \cup\{0\}$ is a Lie algebra and there is a direct sum of modules $\mathfrak{N}=\mathfrak{A}_{S} \oplus\left(\left(\mathfrak{N}-\mathfrak{A}_{S}\right) \cup\{0\}\right)$ Moreover, every non local $m$-derivation of $\mathfrak{N}$ vanishes on $\mathfrak{A}_{s} \subset[\mathfrak{N}, \mathfrak{N}]$. Then $D=D^{11}+D^{21}$ with $D^{11}\left(\right.$ resp. $\left.D^{21}\right)$ the linear component map of $D$ from $\mathfrak{A}_{S}$ to $\langle S\rangle$ (resp. of $\left(\mathfrak{N}-\mathfrak{A}_{S}\right) \cup\{0\}$ towards $\langle S\rangle$ ). By the fact that $D$ is linear and $D^{11}$ takes value in $\langle S\rangle ; D^{11}, D^{21}$ are $m$-derivations.

Proposition 3.15. All m-derivations from $\left(\mathfrak{N}-\mathfrak{A}_{s}\right) \cup\{0\}$ towards $\langle S\rangle$ are Lie derivative with respect to an element of $\langle S\rangle$.

Proof. For $k \in\{1, \ldots, q\}$, we consider the open set $U_{k}=\left\{x \in \mathrm{M} / X_{k}(x) \neq 0\right\}$. We know that all element of $\left(\mathfrak{N}-\mathfrak{A}_{s}\right) \cup\{0\}$ is of the form $C f^{i} X_{j}$ where $C \in \mathbb{R}, f^{i} \in F_{0}(\mathrm{M})$ such that $X_{i}\left(f^{j}\right)$ in $U_{i}$ equals 0 for all $j \neq i$, equals 1 for $j=i$. First, we show that such an $m$-derivation $D$ is local. We fix $i, j$ belonging to $\{1, \ldots, q\}$ with $i \neq j$. Only in $\left\lceil\operatorname{Supp}\left(X_{i}\right)\right.$ we can find an distinguished open set $U$ such that $\left(f^{j} X_{i}\right)_{U} \equiv 0$ or $\left(f^{i} X_{i}\right)_{\mid U} \equiv 0$. It is immediate that $\left[f^{i} X_{i},\left[f^{i} X_{i}, \ldots,\left[f^{i} X_{i}, f^{j} X_{i}\right] \ldots\right]\right]=(-1)^{m-1} f^{j} X_{i}$ by applying $D$ to this last expression, we obtain $\left(D\left(f^{j} X_{i}\right)\right)_{U} \equiv 0$. Now, we let the map $D$ acts on the following bracket $\left[f^{j} X_{j},\left[f^{j} X_{j}, \ldots,\left[f^{j} X_{j}, f^{i} X_{i}\right] \ldots\right]\right]=0$. We have $\left[f^{j} X_{j},\left[f^{j} X_{j}, \ldots,\left[f^{j} X_{j}, D\left(f^{i} X_{i}\right)\right] \ldots\right]\right]_{U} 0$ and $\left(D\left(f^{i} X_{i}\right)\right)_{U} \equiv 0$. In addition, when we are unable to choose $i \neq j$, the proof is trivial. Thus, $D$ is local.

Second, suppose that $D$ is local. Agreeing with the result of Proposition 3.13, we achieve our proof.

Theorem 3.16. All m-derivations of the normalizer $\mathfrak{N}$ of $\mathfrak{A}_{s}$ have a form like the one of Theorem 3.12. Moreover, the normalizer of $\mathfrak{N}$ is $\mathfrak{N}$ itself.

Proof. Given $D=D_{0}+D_{2}$ an $m$-derivation of $\mathfrak{N}$, where $D_{0}$ is local and $D_{2}$ non local. By Proposition 3.10, $D_{0 \mid \mathfrak{A}_{S}}$ is a $m$-derivation of $\mathfrak{A}_{S}$. Let be $X_{1} \in \mathfrak{N}$ and $X_{2}, \ldots, X_{m} \in \mathfrak{A}_{s}$, we write the equation relative to $m$-derivation corresponding to $D_{0}\left[X_{1},\left[X_{2}, \ldots,\left[X_{m-1}, X_{m}\right] \ldots\right]\right]$. By the definition of $\mathrm{m}$-derivations, the previous result and Theorem 3.12, we prove that

$$
\left[\left(D_{0}-\left(\mathrm{L}_{X}+D^{\alpha, \omega}+D_{1}\right)\right)\left(X_{1}\right),\left[X_{2}, \ldots,\left[X_{m-1}, X_{m}\right] \ldots\right]\right]=0
$$

$\forall X_{2}, \ldots, X_{m} \in \mathfrak{A}_{S}$. Let's denote by $D^{\prime}$ the $m$-derivation defined by $D_{0}-\left(\mathrm{L}_{X}+D^{\alpha, \omega}+D_{1}\right), D^{\prime}\left(X_{1}\right)$ belongs to the intersection of the centralizer of $\mathfrak{A}_{S}^{1} \oplus\left[\mathfrak{A}_{S}^{1}, \mathfrak{A}_{S}^{2}\right]$ with $\mathfrak{N}$. With Proposition 3.3, $D^{\prime}\left(X_{1}\right)$ becomes an element of $\langle S\rangle$. In addition, we know that $D_{2 \mid \mathfrak{A}_{S}}=0$. By using $X_{i \neq 1} \in \mathfrak{A}_{S}$ in the relation of $m$-derivation similar to (3.5), the Proposition 3.1 and Proposition 3.3, we have $D_{2}\left(X_{1}\right) \in\langle S\rangle$. Then, Proposition 3.14 and Proposition 3.15 split $D^{\prime}$ to a sum of a derivation of $\mathfrak{A}_{S}$ and $\mathrm{L}_{X}$ with $X \in\langle S\rangle, D_{2}$ is zero. Moreover, we can affirm that the normalizer of $\mathfrak{N}$ coincides with $\mathfrak{N}$ itself.

Proposition 3.17. Every endomorphism $D$ of the commutative
Lie algebra $\langle S\rangle$ is a m-derivation of $\langle S\rangle$. If $D$ is local, it is a Lie derivative with respect to one element of $\mathfrak{N}$. In the case where $D$ is non local, then it is determined by the existence of $i \neq j$ such that $\varnothing \neq\left[\operatorname{Supp}\left(X_{i}\right) \subset \operatorname{Supp}\left(X_{j}\right)\right.$ with $D\left(X_{i}\right)=\lambda_{i}^{k} X_{k}$, where each $\lambda_{i}^{k} \in \mathbb{R}$ and $\lambda_{i}^{j} \in \mathbb{R}^{*}$.

Proof. The first assertion is obvious. Moreover, it is clear that the normalizer of $\langle S\rangle$ is $\mathfrak{N}$, and its centralizer is $\mathfrak{A}_{S}$. Let $D$ be a local endomorphism of $\langle S\rangle$. On $U$, we put $D\left(\frac{\partial}{\partial y^{i}}\right)=\lambda_{i}^{k} \frac{\partial}{\partial y^{k}}$. Then $D=\mathrm{L}_{X}$ with $X \underset{U}{\bar{U}}-\lambda_{i}^{k} y^{i} \frac{\partial}{\partial y^{k}}+Y$ and $Y \in \mathfrak{A}_{S U}$, for all $U$. Thus, $X$ belongs to $\mathfrak{N}$.In addition, if $D^{\prime}$ is a non-local endomorphism of $\langle S\rangle$, let's write $D^{\prime}\left(X_{l}\right)=\lambda_{l}^{k} X_{k}$. It is easy to see that $D^{\prime}$ is non-local iff our last assertion is true.

With the help of the previous proposition, we can confirm immediately

Corollary 3.18. If all elements of $S$ are densely supported over $\mathrm{M}$ or if $S$ is reduced to a singleton, then all endomorphisms of $\langle S\rangle$ is local.

\section{Applications} hold.

The following is a list of some Lie algebras for which our theorems

We denote by $\mathfrak{C}_{c}$ the Lie algebra of all compactly supported vector fields on $\mathrm{M}$ which is an involutive distribution over $\mathrm{M}$. We know that the normalizer of $\mathfrak{C}_{c}$ in $\chi(\mathrm{M})$ is $\chi(\mathrm{M})$ and $O_{\mathfrak{c}_{c}}=\mathrm{M}$ see [7].

We suppose that $\mathrm{M}$ is a differential manifold equipped with a nonsingular generalized foliation $\mathfrak{F}$ see [1]. We denote $\chi(\mathfrak{F})$ (resp. $\chi_{c}(\mathfrak{F})$ the involutive distribution of tangent vector fields to the foliation (resp. of compactly supported vector fields in $\chi(\mathfrak{F})$ ). The normalizer of $\chi(\mathfrak{F})$ in $(\mathrm{M})$ is denoted $\mathfrak{N}(\mathfrak{F})$. The foliation preserving vector fields Lie algebra is named $\mathfrak{L}(\mathfrak{F})$.

Here, $\mathrm{V}$ is a smooth manifold and $\mu$ is surjective smooth map from $\mathrm{M}$ to $\mathrm{V}$ see [5]. It is well known that the set of $\mu$-projected vector fields $\mathcal{N}_{0}$ is a Lie algebra, and $\mu$-zero-projected vector fields set $\eta_{0}$ is an involutive distribution of $\mathrm{M}$. The normalizer of $\eta_{0}$ in $\chi(\mathrm{M})$ is denoted $\mathcal{N}$ and we assume that $O_{\eta_{0}}=M$.

Now, let $\Gamma$ be a connection in the Grifone sense over $M$ cf. [9]. We can cite the curvature horizontal nullity distribution space $\mathfrak{N}_{R}^{h}$ ( $R$ is the curvature), the distribution of horizontal projected vector fields in the curvature nullity space $\mathfrak{A}_{\Gamma}^{h}$. Their respective normalizers in $\chi(\mathrm{TM})$ are designated by $\mathcal{N}_{R}, \mathcal{N}_{\Gamma}$ see [10] and we suppose that $O_{\mathfrak{N}_{R}^{h}}=O_{\mathfrak{2}_{\Gamma}^{h}}=\mathrm{TM}$.

We call $\mathrm{N}^{k}$ the $k$-nullity space distribution of vector fields in the Finsler space considered by Bidabad [11] such that the nullity index doesn't vanish everywhere. Let's note that $\mathcal{N}^{k}$ is its normalizer in the vector fields Lie algebra.

Thus, replacing respectively $\mathfrak{A}$ by $\chi(\mathrm{M}), \mathfrak{C}_{c}, \chi(\mathfrak{F}), \chi_{c}(\mathfrak{F})$, $\mathfrak{L}(\mathfrak{F}), \eta_{0}, \mathcal{N}_{0}, \mathfrak{N}_{R}^{h}, \mathfrak{A}_{\Gamma}^{h}, \mathrm{~N}^{k} \quad$ and $\mathfrak{B} \quad$ by $\quad \chi(\mathrm{M}), \quad \chi(\mathrm{M})$, $\mathfrak{N}(\mathfrak{F}), \mathfrak{N}(\mathfrak{F}), \mathfrak{N}(\mathfrak{F}), \mathcal{N}, \mathcal{N}, \mathcal{N}_{R}, \mathcal{N}_{\Gamma}, \mathcal{N}^{k}$; we state that "All $m$-derivation of $\mathfrak{A}$ (resp. of $\mathfrak{B}$ ) is inner with respect to $\mathfrak{B}$ (resp. is inner)". 
Citation: Randriambololondrantomalala P (2015) The $m$-Derivations of Distribution LieAlgebras. J Generalized Lie Theory Appl 9: 217. doi: 10.4172/1736-4337.1000217

In addition, let's consider the system $S$ composed by the Liouville vector field $C$ on TM. We work on TM without zero section, we find all $m$-derivation of $\mathfrak{A}_{S}$ by our theorem, as well as its normalizer which is locally isomorphic to $\mathfrak{A}_{S} \oplus g l(1, \mathbb{R})$. By density of the foliation regular points set defined by $S$, we obtain analogous results on TM. All $\mathbb{R}$ -linear maps of $\langle C>$ into itself are local.

The author benefits an Ingrid Daubechies initiative scholarship in collaboration with" Institute for the Conservation of Tropical Environments" (ICTE) Madagascar.

\section{References}

1. Randriambololondrantomalala $P$ (2014) Lie derivations m-algebras of polynomial vector fields, Submitted to Africa Mathematics.

2. Chen $\mathrm{C}$, Lian $\mathrm{H}(2012) \mathrm{N}$-derivations for finitely generated graded Lie algebras. arXiv $12060309 \mathrm{v} 1$.

3. Peetre J (1960) Corrections to the article "An abstract characterization of differential operators". Math Scand 8: 116-120.
4. Ravelonirina HSG, Randriambololondrantomalala P, Anona M (2012) The Lie algebra of a system of vector fields swappable. Italian Journal of Pure and Applied Mathematics-N 29: 163-174.

5. Randriambololondrantomalala $P(2010)$ The Lie algebra of a distribution and a generalized foliation. PhD Thesis, University of Antananarivo, Madagascar.

6. Lecomte $P$ (1981) On the infinitesimal automorphism of the vector bundle. J Math Pures Appl 60: 229-239.

7. Randriambololondrantomalala P, Ravelonirina HSG, Anona M (2010) The Lie algebra of a distribution and a generalized foliation. African Diaspora Journal of Mathematics 2: 135-144.

8. Zhou JH (2013) Triple derivations of perfect Lie algebras. Comm. Algebras 41: 1647-1654.

9. Grifone J (1972) Almost tangent structure and Connections I. Ann Inst Fourier 22: $287-334$.

10. Randriambololondrantomalala P, Ravelonirina HSG, Anona M (2015) On Lie algebras associated with a connection.

11. Bidabad B, Rafie-Rad M (2011) On the k-nullity foliations in Finsler space. Bulletin of the Iranian Mathematical Society 37: 1-18. 